\title{
Collaborative Filtering with Short Term Preferences Mining
}

\author{
Diyi Yang, \\ Tianqi Chen, \\ Weinan Zhang, \\ Shanghai Jiao Tong University \\ 800 Dongchuan Road, Shanghai China \\ \{yangdiyi, tqchen, wnzhang,yyu\}@apex.sjtu.edu.cn \\ Yong Yu
}

\begin{abstract}
Recently, recommender systems have fascinated researchers and benefited a variety of people's online activities, enabling users to survive the explosive web information. Traditional collaborative filtering techniques handle the general recommendation well. However, most such approaches usually focus on long term preferences. To discover more short term factors influencing people's decisions, we propose a short ter$\mathrm{m}$ preferences model, implemented with implicit user feedback. We conduct experiments comparing the performances of different short term models, which show that our model outperforms significantly compared to those long term models.
\end{abstract}

\section{Categories and Subject Descriptors}

H.3.3 [Information Systems]: Information Search and Retrieval-Information Filtering

\section{General Terms}

Algorithms, Models, Experiments

\section{Keywords}

Collaborative Filtering, User Feedback, Recommender Systems

\section{INTRODUCTION}

Collaborative filtering (CF) has been widely used in web services, such as products recommendation at Amazon $^{1}$ and music recommendation at Pandora ${ }^{2}$. There are two canonical approaches to attack this problem. Latent factor model$\mathrm{s}$, like singular value decomposition (SVD), map users and items into the same low-dimensional space and predict users' interest on items based on the relations between mapped vectors. The second are neighborhood models, which concentrate on detecting the correlations or similarities between items/users. Both approaches focus on users' long term interests, and do recommendation based on those stable preferences.

However, people's interests are likely to change over time. Long term interests, stay stable for a long time, but short

\footnotetext{
${ }^{1}$ http://www.amazon.com/

${ }^{2}$ http://www.pandora.com
}

Copyright is held by the author/owner(s).

SIGIR'12, August 12-16, 2012, Portland, Oregon, USA.

ACM 978-1-4503-1472-5/12/08. term preferences are volatile. They may depend more on the current situation, users' mood at present, or his/her recent actions. For example, John might suddenly change from his favorite Jazz to light songs just because he had an unexpected guest who required it. It is hard to capture, but better performances will be achieved if considered. We extract this preference, and integrate it with the classical SVD++ model. Experiments conducted on Yahoo! Music dataset show that our model achieves better performances than the baseline SVD++, which proves that short term information does help capture users' changing preferences. We present our models in Section 2, and experimental results in Section 3. In Section 4, we conclude our work.

\section{SHORT TERM MODELS}

We introduce our models for short term preferences mining in this section, and characterize short term property through users' implicit feedback. Implicit feedback, i.e. the users' rating history, as an indicator of user preferences, refers to all kinds of behaviors that users have performed on items. The traditional matrix factorization model with implicit feedback, called SVD ++ , predicts user $u$ 's rating $\widehat{r}_{u i}$ on item $i$ as follows[2]:

$$
\widehat{r}_{u i}=b_{u}+b_{i}+\left(p_{u}+\frac{\sum_{j \in N(u)} y_{j}}{\sqrt{|N(u)|}}\right)^{T} q_{i}
$$

Here, $N(u)$ is the item set of implicit feedback of user $u$. $b_{u}$ is the users' bias and items' bias denotes as $b_{i}$. Each user $u$ is related to $p_{u}$, and each item $i$ are associated with vectors $q_{i}, y_{j}\left(p_{u}, q_{i}, y_{j} \in \mathbb{R}^{k}\right)$.

\subsection{Incorporate Short Term Implicit Feedback}

Even though global implicit feedback performs effectively, it only captures users' long term preferences by using the users' whole rating history. Since users' interests vary with time, we also need to take the short term, especially the current interest, into consideration. Therefore, we propose a novel short term model as follows:

$$
\widehat{r}_{u i}(t)=b_{u}+b_{i}+\left(p_{u}+\frac{\sum_{j \in N(u)} y_{j}}{\sqrt{|N(u)|}}+\frac{\sum_{j \in N(u, t)} \xi_{j}}{\sqrt{|N(u, t)|}}\right)^{T} q_{i}
$$

Here $N(u, t)$ is user $u$ 's short term implicit feedback during time interval $t$. $t$ is a time indicator that could be week, day, hour, or even minute. $\xi_{j}\left(\in \mathbb{R}^{k}\right)$ is an item revelent vector. By limiting the original global implicit feedback to a short time $t$ like a minute/an hour, it can capture those fac- 


\begin{tabular}{|c|c|}
\hline Models & Average RMSE \\
\hline \hline GSVDP & 23.233 \\
\hline Clock.GSVDP & $\mathbf{2 2 . 6 7 3}$ \\
\hline Hour.GSVDP & 22.814 \\
\hline Day.GSVDP & 22.922 \\
\hline Week.GSVDP & 22.972 \\
\hline
\end{tabular}

Table 1: RMSE of different short term implicit feedback models

tors that influences people's decisions directly. For example, an Elsevier ${ }^{3}$ user may start to download some CF relevant papers because of confusion in recommendation techniques that a biological paper he is reading refers to. This model captures users' short term interests through their behaviors during a certain period, and does not require extra parameters when we need $t$ to be finer granularity, differing from the traditional temporal dynamics model[3].

\section{EXPERIMENT}

\subsection{Experiment Setup}

We use the Yahoo! Music Track 1 dataset[1] introduced in the KDDCup 2011. It contains about 250 million ratings rated by 1 million users for about 0.6 million items with time stamps. We use the official training and validation dataset for training, and report RMSE (Rooted Mean Square Error) on test dataset. To investigate the effectiveness of short term implicit feedback, we utilize five kinds of information, whose implicit feedback comes from the whole history, current week, current day, current hour and current minute(the least time granularity in dataset). We call the model which uses all of the users' rating history as GSVDP. The others are Week.GSVDP, Day.GSVDP, Hour.GSVDP and Clock.GSVDP. Moreover, since we want to test whether the short term preferences help enhance GSVDP, those four models are all implemented with GSVDP as Equation 2. As well as comparing overall performance of the five version$\mathrm{s}$, we also discuss the resolution at which time granularity contributes most. Finally, to further analyze the consistency of short term preferences, we conduct experiments and present each category's differences between GSVDP and Clock.GSVDP.

\subsection{Experiment Results}

The results of our experiments are presented in Table 1. We find that short term implicit feedback models outperform basic GSVDP significantly. From GSVDP's 23.233 to each short term models' $22 . x$, different degree of improvements have been given. For example, Clock.GSVDP gives 22.673, achieving the greatest improvement among all the short term models. The runner up result is given by Hour.GSVDP(22.814). Week.GSVDP produces 22.972, and Day.GSVDP is a little better than week model with a RMSE of 22.922. Therefore, it is reachable that short term implicit feedback models assure big improvement overall in contrast to GSVDP.

It is naturally discovered that as time granularity increases, short term models give less satisfying performances. Since Clock.GSVDP is superior to Hour.GSVDP, and Hour.G-

\footnotetext{
${ }^{3}$ www.elsevier.com
}

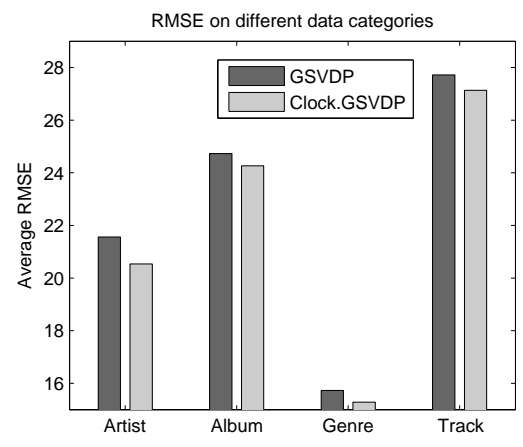

Figure 1: Performance Comparison on Different Categories

SVDP outperforms Day.GSVDP with day model superior to Week.GSVDP, we consider that relative large time granularity cannot preserve the short term property, i.e. latest users' activities affects their future's decisions powerfully. Week model performs the nearly same RMSE as day model, that is, when reaching certain time granularity, models forfeit the ability of precisely capturing such preferences. Thus, clock short term implicit feedback is more predictive than hour, day and week granularity, consisting with our experimental results. Implemented with GSVDP which captures the users' long term preferences, our short term models have both long term and short term capabilities to fully capture users' interest.

To investigate whether the short term model produces the consistency of improvement or not, we present each category's RMSE changes between GSVDP and Clock.GSVDP in Figure 1. There are four categories, artist, album, genre and track, in Yahoo! Music dataset. As we can see, short term implicit feedback models give decreased RMSE on all categories. This consistency of improvement emphasizes again that mining short term information will do well in enhancing a model's overall performance.

\section{CONCLUSION}

In this work, we extract the short term preferences to enhance our model, and implemented experiments are conducted in different short term settings. We conclude that short term performs well in capturing users' perdue interests and predicts changing preferences more precisely. In the future, we are determined to explore short term preferences further and improve our model systematically.

\section{ACKNOWLEDGEMENT}

Yong $\mathrm{Yu}$ is supported by grants from NSFC-RGC joint research project 60931160445 .

\section{REFERENCES}

[1] G. Dror, N. Koenigstein, Y. Koren, and M. Weimer. The yahoo! music dataset and kdd-cup'11. In KDD-Cup Workshop 2011, 2011.

[2] Y. Koren. Factorization meets the neighborhood: a multifaceted collaborative filtering model. In Proceeding of the 14th ACM SIGKDD international conference on Knowledge discovery and data mining, KDD '08, 2008.

[3] Y. Koren. Collaborative filtering with temporal dynamics. In Proceedings of the 15th ACM SIGKDD international conference on Knowledge discovery and data mining, KDD '09, 2009. 